\title{
Author's Response to: Letter to Editor
}

\author{
Michael K. O'Reilly ${ }^{1}$ ' Gavin Sugrue ${ }^{1}$ Cormac T. Farrelly ${ }^{1}$
}

Received: 28 March 2017/ Accepted: 14 April 2017/Published online: 5 May 2017

(C) Springer Science+Business Media New York and the Cardiovascular and Interventional Radiological Society of Europe (CIRSE) 2017

To the editor,

Thank you to Conroy et al. for their interesting letter. This certainly provides further evidence supporting the safety and efficacy of pneumatic compression devices (PCD) for achieving haemostasis in patients undergoing haemodialysis fistula intervention. Their series included 116 device deployments in an unstated mixture of arteriovenous fistulas and grafts, and so, it would also appear to support its use after accessing dialysis grafts and with sheath sizes up to 7 Fr.

It is interesting that both case series examining this use of achieving haemostasis used the same PCD, namely the Safeguard Radial Compression Device (Merit Medical Systems Inc. Utah, USA). This is a 26-cm-long self-adhesive band that is designed for achieving patent haemostasis after radial artery catheterisation. We believe it is important to point out that not all radial artery PCDs are the same. For example, other PCDs incorporate nonadhesive wrist bands that are required to fit completely around the wrist (similar to a watch) and may have a larger surface area. Where a wrist band may be expected to work at a radiocephalic Cimino-Brescia-type fistula, if the band is not large enough to fit around the elbow it will not be possible to use it on a brachiocephalic or brachiobasilic-

Michael K. O'Reilly

Moreilly1@mater.ie

1 Department of Interventional Radiology, Mater Misericordiae University Hospital, Eccles St., Dublin 7, Ireland type fistula or indeed many arteriovenous grafts. Also PCDs with a larger surface area may make it more difficult to achieve haemostasis at two adjacent fistula/graft catheterisation access sites.

One of the advantages of using a PCD is the ability to accurately control the amount of compression at the access site. This allows optimal compression without occlusion. The concept of patent haemostasis is now well established with transradial artery access, and indeed, there is evidence that a decrease in radial artery occlusion after radial artery catheterisation can result from prophylactic ipsilateral ulnar artery compression [1]. Although a dialysis fistula has a significantly larger lumen than the radial artery, it would be interesting to study whether similar benefits in radiocephalic fistula patency rate could be achieved with concomitant ulnar artery compression.

Regardless, further studies are warranted looking at the use of various types of PCDs for haemostasis and ideally manufacturers would design PCDs specifically for use after dialysis access intervention. In the interim, we continue to use the PCD described in our study and we would advise our colleagues to join us with a technique that is simple, speedy, safe and sutureless.

\section{Compliance with Ethical Standards}

Conflict of interest The authors declare that they have no conflict of interest.

\section{Reference}

1. Pancholy SB, Bernat I, Bertrand OF, Patel TM. Prevention of radial artery occlusion after transradial catheterization: the prophet-II randomized trial. JACC Cardiovasc Intervent. 2016;9(19):1992-9. 